\title{
Epidemiologia da disfunção temporomandibular: uma revisão de literatura
}

\author{
Epidemiology of temporomandibular dysfunction: a literature review
}

\author{
Karine Renatta Barros Góes', Manassés Tercio Vieira Grangeiro², \\ Viviane Maria Gonçalves de Figueiredo ${ }^{3}$
}

'Centro Universitário Leão Sampaio - Juazeiro do Norte, Ceará - Brasil. karinegoes@gmail.com ${ }^{2}$ Autor para correspondência. Universidade Estadual Paulista - São José dos Campos, São Paulo - Brasil. terciomanasses@gmail.com ${ }^{3}$ Centro Universitário Leão Sampaio - Juazeiro do Norte, Ceará - Brasil. vivi_mfigueiredo@yahoo.com.br

RESUMO I INTRODUÇÃO: A disfunção temporomandibular (DTM) pode estar presente, em média, em $50 \%$ da população onde apresentam algum sinal ou sintoma da patologia. OBJETIVO: revisar a epidemiologia da disfunção temporomandibular na literatura; com ênfase nas seguintes variáveis: gênero, faixa etária e sinais e sintomas. MATERIAL E MÉTODO: As bases de dados utilizadas foram Pubmed, Scielo, Bireme e Lilacs em que foram coletados artigos através das palavras-chaves que foram: Epidemiologia; Transtornos da Articulação Temporomandibular; Sinais e Sintomas. Já os critérios de inclusão apresentados foram: literatura que aborde a temática em estudo, literatura dos últimos anos, estudos epidemiológicos e clínicos, revisão sistemática, literatura em português e inglês. Enquanto que os critérios de exclusão foram: carta ao editor, artigo de opinião, literatura duplicada em base de dados, por fim literatura que não apresenta as variáveis estudadas. RESULTADO: A epidemiologia da disfunção temporomandibular apresenta-se muito prevalente em populações distintas, como idosos, adolescentes e principalmente em adultos jovens. CONCLUSÃO: Os sinais e sintomas mais frequentes são dor muscular, dor articular, estalido e tensão emocional.

PALAVRAS-CHAVE: Epidemiologia. Transtornos da Articulação Temporomandibular. Sinais e Sintomas.

\begin{abstract}
INTRODUCTION: Temporomandibular disorder (TMD) may be present in the media in $50 \%$ of the population in any sign or symptom of the condition. OBJECTIVE: To review an epidemiology of temporomandibular dysfunction in the literature; With emphasis on the various variables gender, age range and signs and symptoms. MATERIAL AND METHOD: As databases use Pubmed, Scielo, Bireme and Lilacs. As keywords for a textual search were Epidemiology. Temporomandibular Joint Disorders. Signals and symptons. The inclusion criteria were published in a scientific journal, literature on the last years, epidemiological and clinical studies, systematic review, literature in Portuguese and English. While the exclusion criteria for literature review, letter to the editor, opinion article, literature duplicated in the database, finally literature that does not present as studied variables. RESULT: The epidemiology of temporomandibular dysfunction is very prevalent in distinct populations, such as the elderly, adolescents and especially in young adults. CONCLUSION: Just as the most frequent signs and symptoms are muscle pain, joint pain, cracking and emotional tension.
\end{abstract}

DESCRIPTORS: Epidemiology. Temporomandibular Joint Disorders. Signals and symptons. 


\section{Introdução}

Em média 50 a $60 \%$ da população apresenta algum sinal ou sintoma de Disfunção Temporomandibular (DTM), uma doença relacionada ao mau funcionamento do sistema estomatognático, promovendo dores musculares, articulares, de ouvido, dores de cabeça entre outros'. Ansiedade e o estresse são fatores emocionais que podem desencadear hábitos parafuncionais e tensão muscular, auxiliando no aparecimento dos sinais e sintomas das DTMs². Os fatores etiológicos das DTMs estão diretamente relacionados a fatores oclusais, neuromusculares e emocionais, podendo apresentar-se de forma associada ou nao ${ }^{3}$. O aumento do número de novos casos das dores orofaciais crônicas referente às DTMs e suas repercussões na qualidade de vida das pessoas, têm merecido relevância nas investigações em saúde pública ${ }^{4}$.

O bruxismo esta entre os fatores com maior destaque na DTM, pois é um ato automático, parafuncional, de comprimir os dentes, esfregar ou ranger, obtendo assim um desgaste na estrutura dentária, como também podendo estar diretamente ligado ao estresse e á ansiedade 5 .

No serviço odontológico oferecido pela UNICAMP, notou-se um aumento de procura pelo tratamento da DTM no decorrer dos anos, acerca do tempo moderno juntamente com fatores que desencadeiam esta patologia bem como a ansiedade, estresse, depressão, fadiga, insônia e dores crônicas ${ }^{6}$. A dor crônica proveniente da DTM é também influenciada pelo estado psicológico dos pacientes, o que causa grande influência no comportamento social dos mesmos. ${ }^{7} \mathrm{O}$ impacto da qualidade de vida dos pacientes é grande quando eles recebem orientações sobre as causas da DTM, os hábitos a serem modificados, postura correta para dormir, trabalhar, estudar, como também sobre o estresse que é o fator principal ou fator que aumenta significativamente a sintomatologia dos pacientes ${ }^{6}$.

A DTM apresenta etiologia multifatorial, podendo destacar fatores predisponentes, desencadeantes, e perpetuantes, que podem ser: psicogênicos, mecânicos, bio-psicossociais, parafuncionais e múltiplos hábitos parafuncionais ${ }^{8}$. Essa patologia é muito pre- valente na população, principalmente em adultos jovens com faixa etária entre 20 e 40 anos?.

Assim se faz necessário investigar a epidemiologia desta disfunção em populações distintas, a fim de verificar a presença da mesma, bem como indicar a terapêutica necessária para estes indivíduos com DTM. De acordo com o exposto anteriormente, objetiva-se revisar a epidemiologia da disfunção temporomandibular na literatura; com ênfase nas seguintes variáveis gênero, faixa etária e sinais e sintomas.

\section{Revisão de liłeratura}

\section{Seleção dos Estudos}

Esta revisão de literatura foi conduzida por meio das seguintes bases de dados: Pubmed (https:// www.ncbi.nlm.nih.gov/pubmed), Scielo (http://www. scielo.org/php/index.php), Bireme (http://bvsalud. org/) e Lilacs (http://lilacs.bvsalud.org/).

As palavras-chave para a busca textual foram: "Epidemiologia", "Transtornos da Articulação Temporomandibular";" Disfunção da Articulação Temporomandibular, "Sinais e Sintomas". Já os critérios de inclusão foram: Literatura que aborde a temática em estudo, Literatura dos últimos anos, Idioma Inglês e Português, estudos epidemiológicos e clínicos e revisão sistemática. Os critérios de exclusão foram: revisão de literatura, carta ao editor, artigo de opinião, literatura duplicada em bases de dados e literatura que não abordasse as variáveis em estudo.

\section{Histórico}

Em 1934, a DTM foi descrita como síndrome de Costen, a qual foi descoberta pelo médico otorrinolaringologista James Costen após alguns relatos dos seus pacientes sobre dores na região auricular e pré auricular ${ }^{12}$.

Em 1959, Shore modificou a nomenclatura para síndrome da disfunção da ATM, logo após Ramiford e Ash introduziram o termo síndrome da disfunção da ATM, já Bell propôs o termo desordens tempo- 
romandibulares. Por fim a ADA (American Dental Association) adotou o termo DTM ou desordem temporomandibular ${ }^{12}$.

\section{Conceito DTM}

A DTM é uma patologia que engloba músculos da mastigação, a Articulação Temporomandibular (ATM) e estruturas vizinhas. Como também, o indivíduo pode apresentar dificuldades ao abrir a boca, dor na região auricular e pré-auricular, ruídos e dores na ATM e nos músculos da mastigação. Por fim, a dor pode ter etiologia muscular ou articular, bem como ocorrerem em conjunto ${ }^{10,11}$.

Esta desordem é o conjunto de sinais e sintomas que podem se manifestar nos músculos da mastigação, ATMs, bem como também em outras estruturas do sistema estomatognático ${ }^{8}$. A DTM pode ser classificada como um termo coletivo que abrange alguns problemas clínicos que afetam os músculos da mastigação, a ATM e estruturas vizinhas ${ }^{12}$.

\section{Etiologia}

A etiologia da DTM é multifatorial, pois se relaciona com modificações na oclusão, traumatismos e desgastes da ATM, hábitos parafuncionais, problemas psicológicos e complicações esqueléticas ${ }^{10}$.

Os fatores oclusais não devem ser classificados os mais importantes na etiologia das DTMs. É de suma importância uma visão ampla dos seus fatores etiológicos e reconhecer a oclusão como apenas mais um desses fatores, podendo esta atuar em maior ou menor grau, dependendo das características do paciente $^{13}$.

\section{Sinais e Sintomas}

A DTM caracteriza-se pela presença de sinais e sintomas que podem surgir coincidentemente, como também a queixa de dificuldades ou desconforto no decorrer da fala que é observada e relatada por indivíduos com DTM ${ }^{14}$.

Os sinais e sintomas são amplos e diversos, e podem se apresentar com dor na região da ATM e na região auricular, fadiga muscular, ruídos, dificuldade em abrir a boca, estalos acompanhados de dor, dor de cabeça, e esses sintomas são agravados quando em função mastigatória e/ou mandibular ${ }^{11}$.

Diante a história e o exame clínico, encontram-se as mais diversas queixas como dor cervical ou facial, movimentos mandibulares bem limitados, ruídos na ATM, sensações incomuns na oclusão e na cavidade oral e, por vezes também, alterações na audição, equilíbrio, visão ou até queixa de mal-estar podem ser relatadas ${ }^{1}$.

\section{Epidemiologia}

A DTM tem sua maior prevalência entre 20 e 45 anos, entretanto até os 40 anos, a principal causa é de origem muscular, que nomeamos de DTM miogênica ou muscular já a partir dos 40 anos, o principal fator etiológico é a degeneração articular, que nomeamos DTM artrogênica ou articular ${ }^{9,11}$.

Alguns estudos mostram maior prevalência da disfunção temporomandibular no sexo feminino. Fatores anatômicos como frouxidão ligamentar e alterações hormonais ligados ao ciclo menstrual também foram estudados no intuito de justificar a prevalência pelo sexo feminino ${ }^{15}$.

A prevalência de sinais e sintomas de DTM em pacientes que frequentam o Serviço de Controle da Dor Orofacial do Departamento de Odontologia da Universidade Estadual da Paraíba, foi de $80 \%$ em paciente com faixa etária entre 20 e 40 anos $^{11}$.

Já em estudantes universitários a prevalência dos sinais e sintomas de DTM foi de $73,7 \%$ nos indivíduos do sexo feminino com faixa etária entre 21 anos em média e de $26,3 \%$ nos indivíduos do sexo masculino com faixa etária entre $22 \operatorname{anos}^{16}$.

Em outro estudo, 20\% dos idosos que foram avaliados apresentavam algum sinal ou sintoma de DTM, sendo $10 \%$ do sexo feminino e $90 \%$ do sexo masculino, encontrando-se numa faixa etária de 60 a 90 anos $^{17}$.

Foi analisado a prevalência de pacientes com presença de DTM que foram atendidos entre 2005 -2011 no Ambulatório do Serviço de Controle da 
Dor Orofacial do Hospital Universitário Lauro Wanderley da Universidade Federal da Paraíba, em João Pessoa, que mostrou uma preponderância pelo sexo feminino $(80 \%)$ e ao utilizar um questionário como veículo para descoberta das DTMs, obteve-se um resultado de $64 \%$ de pacientes com sinais e sintomas de DTM ${ }^{18}$.

Em outro estudo, foi coletado uma amostra de 732 universitários do curso de Fisioterapia de uma Universidade de Manaus, com faixa etária entre 18 e 54 anos, onde a prevalência de DTM foi de $(79,9)$ para o sexo feminino $(79,9)$ e $(20,1)$ no sexo masculino ${ }^{19}$.

Uma determinada população de policiais militares foi abordada para identificar a prevalência de DTM entre eles. Estudo realizado com 148 indivíduos com idade de 18 a 36 anos, onde houve presença de DTM em $49,3 \%$ dos pesquisados, a prevalência foi maior no sexo feminino ${ }^{20}$.

A DTM é uma condição com alta prevalência em pacientes com cefaleias primárias $(54,8 \%)$, apresentando mais de 6 sintomas, onde a prevalência foi maior no sexo feminino com resultado de $64 \%$, onde a média de idade dos entrevistados era entre 31 anos $^{21}$.

Em outro estudo não houve associação estatisticamente significativa entre DTM e sexo ou classe socioeconômica, no entanto houve associação estatisticamente significativa com a idade ${ }^{22}$.

Outro estudo que foi constituído de 200 idosos, apresentou um significante número de DTM (61\%), onde $\circ$ sexo feminino se mostrou mais prevalente com $(72,4 \%)$ e o masculino com $(41,1 \%)^{23}$.

Um estudo que teve como amostra 580 estudantes universitários mostra que a prevalência de sinais e sintomas de DTM no sexo feminino foi de (14\%) e o sexo masculino de $(16 \%)$ onde se fizeram presente no estudo 66 mulheres e somente 18 homens, sendo assim a prevalência no sexo feminino foi um número mais considerável que o sexo masculino ${ }^{24}$.

Existem variados meios de avaliação da DTM que devem ser selecionados de acordo com o paciente e o profissional que está realizando. Por meio da necessidade de se avaliar o impacto das DTMs na qualidade de vida, foram surgindo alguns métodos para isso. Os índices são realizados para rastrear sinais e até sintomas da DTM viabilizando a gravidade da disfunção. Já os questionários são utilizados geralmente para descobrir os sintomas presentes, podendo ser realizado de diversas maneiras, sendo elas em contato direto com quem está sendo entrevistado ou não. Os meios de avaliação são citados como: Índice craniomandibular (ICM), Índice de Helkimo, Questionário da Academia Americana de Dor Orofacial, Questionário de Limitação Funcional Mandibular (MFIQ) e o Questionário e Índice Anamnésico de Fonseca ${ }^{25}$.

O Índice Anamnésico de Fonseca foi elaborado a partir dos índices anamnésicos de Helkimo, no qual, depois de testado, mostrou-se correlacionar bastante com $\circ$ índice de Helkimo. Este questionário é composto por três respostas em cada pergunta, onde cada uma tem sua pontuação distinta. É um dos questionários mais utilizados nos dias atuais por ser simples para realizar, onde vai qualificar a severidade da disfunção, todavia consiste de algumas limitações como a falta de classificação diagnóstica da DTM e sua pontuação também não se mostra tão específica quanto desejado ${ }^{25}$.

Quanto aos sinais e sintomas, a prevalência dentre os estudos revisados foram observados nos resultados que $14 \%$ dos entrevistados apresentavam episódios de dores miofasciais ${ }^{22}$. Já outro estudo, 78, 1 $\%$ apresentaram percepção dolorosa nos músculos mastigatórios e $77,2 \%$ nos músculos cervicais ${ }^{23}$. Outro estudo foi baseado em pacientes com algum tipo de dor de cabeça primária, fazendo assim ser o sintoma mais prevalente entre os indivíduos presentes 21. O transtorno mais corriqueiro foi o estalido na ATM, onde esteve presente em $7 \%$ dos alunos ${ }^{24}$. Cada região anatômica facial dispõe de diversas prevalências de sinais e sintomas ${ }^{16}$. Outro estudo foi observada a prevalência de tensão emocional, onde $84 \%$ dos indivíduos relataram a presença da mesma, com variação de intensidade de $\mathrm{Oa} 10$ a avaliação $^{18}$. A dor articular foi a queixa que mais prevaleceu com $45 \%$ dos casos, e no que se refere aos sinais e sintomas o ruído esteve presente em $95 \%$ dos $\operatorname{casos}^{11}$. No estudo que utilizou de um questionário no qual não obtinha cálculo de escores, foi proposto como principal sinal de DTM, a existência 
de estalos ou cliques durante o ato de elevação ou depressão da mandíbula ${ }^{17}$. Já outro estudo os sinais e sintomas que mais se fizeram presentes foram o fato das pessoas se sentirem tensas ou nervosas com $71,6 \%$ dos $\operatorname{casos}^{20}$.

Novos estudos devem ser realizados para favorecer a discussão dos sinais e sintomas, como também prevalência desta disfunção na população. Pois, os estudos são realizados com populações muito distintas o que dificulta a observação destes sinais e sintomas.

\section{Conclusão}

A epidemiologia da disfunção temporomandibular apresenta-se muito prevalente em populações distintas, como idosos, adolescentes e principalmente em adultos jovens. Assim como os sinais e sintomas mais frequentes são dor muscular, dor articular, estalido e tensão emocional.

\section{Contribuição dos autores}

Góes KRB, participou da etapa de conceitos e ideias para a pesquisa em questão, confecção do projeto, definição do tema intelectual, busca bibliográfica. Grangeiro MTV, participou da etapa de busca bibliográfica, preparação, revisão e edição do manuscrito. Figueiredo $V M G$, participou da etapa de conceitos e ideias para a pesquisa em questão, confecção do projeto, definição do tema intelectual, preparação, revisão e edição do manuscrito.

\section{Conflitos de interesses}

Nenhum conflito financeiro, legal ou político envolvendo terceiros (governo, empresas e fundações privadas, etc.) foi declarado para nenhum aspecto do trabalho submetido (incluindo mas não limitandose a subvenções e financiamentos, conselho consultivo, desenho de estudo, preparação de manuscrito, análise estatística, etc).

\section{Referências}

1. Okeson JP. Tratamento das desordens temporomandibulares e oclusão. 4.ed. São Paulo: Artes Médicas; 2000.
2. Garcia AR, Martins RJ, Garbin CAS, Zuim PRJ, Sundefeld MLMM. Fatores associados à ocorrência de vibrações articulares. Rev Fac Odontol Porto Alegre. 2009;50(1):24-28.

3. Felício CM. Fonoaudiologia aplicada aos casos odontológicos: motricidade oral e audiologia. São Paulo: Pancast; 1999.

4. Barbosa TS, Miyakoda LS, Pocztaruk RL, Rocha CP, Gavião $M B D$. Temporomandibular disorders and bruxism in childhood and adolescence: review of the literature. Int $\mathrm{J}$ Pediatr Otorhinolaryngol. 2008;72(3):299-314. doi: 10.1016/i. iiporl.2007.11.006

5. Cestari K, Camparis CM. Fatores psicológicos: sua importância no diagnóstico das desordens temporomandibulares. J Bras Oclusão, ATM \& Dor Orofacial. $2002 ; 2(5): 54-60$.

6. Moreira APSM, Cruvinel LL, Bortolleto PPB. Programa preventivo para dor orofacial e disfunção das articulações temporomandibulares (ATM). Sínteses: Revista Eletrônica do SIMTEC. $2016 ; 2: 308$. doi: 10.20396/sinteses.v0i2.8814

7. Seger L. Psicologia e odontologia: Uma abordagem integradora. 4.ed. São Paulo: Santos; 2002.

8. Paiva HJ. Noções e conceitos básicos em oclusão, disfunção temporomandibular e dor orofacial. São Paulo: Livraria Santos Editora; 2008.

9. Tosato JP, Caria PHF. Prevalência de DTM em diferentes faixas etárias. RGO. 2006;54(3):211-224.

10. Donnarumma MDC, Muzilli CA, Ferreira C, Nemr K. Disfunções temporomandibulares: sinais, sintomas e abordagem multidisciplinar. Revista CEFAC. 2010;12(5):788794. doi: $\underline{10.1590 / \text { S1516-18462010005000085 }}$

11. de Figueiredo VMG, Cavalcanti AL, Farias $A B L$, Nascimento SR. Prevalência de sinais, sintomas e fatores associados em portadores de disfunção temporomandibular. Acta Scientiarum. Health Sciences. 2009;31 (2):159-163. doi: 10.4025 /actascihealthsci.v31 i2.5920

12. Okeson JP. Tratamento das desordens temporomandibulares e oclusão. 6.ed. Rio de Janeiro: Elsevier; 2008.

13. Lemos GA, Moreira VG, Forte FDS, Beltrão RTS, Batista AUD. Correlação entre sinais e sintomas da Disfunção Temporomandibular (DTM) e severidade da má oclusão. Rev Odontol UNESP. 2015;44(3):175-80. doi: 10.1590/1807. $\underline{2577.1084}$

14. Taucci RA, Bianchini EMG. Verificação da interferência das disfunções temporomandibulares na articulação da fala: queixas e caracterização dos movimentos mandibulares. Rev Soc Bras Fonoaudiol. 2007; 1 2(4):274-80. doi: 10.1590/ $\underline{\text { S1516-80342007000400004 }}$ 
15. Martins RJ, Garcia AR, Garbin CAS, Sundefeld MLMM. Relação entre classe socioeconômica e fatores demográficos na ocorrência da disfunção temporomandibular. Ciênc Saúde Coletiva. 2008;13(2):2089-2096. doi: 10.1590/S1413$\underline{81232008000900013}$

16. Oliveira AS, Bevilaqua-Grossi D, Dias EM. Sinais e sintomas da disfunção temporomandibular nas diferentes regiões brasileiras. Fisioterapia e Pesquisa. 2008; 15(4):392396.

17. Carvalho KC, Lélis EM, Carvalho NF, Ferreira DLA, Rocha GM, Souza APS et al. Prevalência dos sinais e sintomas sugestivos de disfunções temporomandibulares em um grupo de idosos da cidade de Teresina. ConScientiae Saúde. 2010;9(3):441-447.

18. Dantas AMX, Santos EJL, Vilela RM, Lucena LBS. Perfil epidemiológico de pacientes atendidos em um Serviço de Controle da Dor Orofacial. 2015;44(6):313-319. doi: 10.1590/1807-2577.1065

19. Pinto AL, Gomes Júnior VFF, Mesquita CM, Ripardo ECN, Silva EF, Penalber GML et al. Prevalência da disfunção temporomandibular e qualidade de vida em acadêmicos de Fisioterapia. J Health Sci Inst. 2015;33(4):371-375.

20. Cavalcanti MOA, Lima JMC, Batista AUD, Oliveira LMC, Lucena LBS. Grau de severidade da disfunção temporomandibular e hábitos parafuncionais em policiais militares. RGO - Rev Gaúcha de Odontol. 201 1;59(3):351 356.

21. Tomaz-Morais JF, Lucena LBS, Mota IA, Pereira AKFTC, Lucena BTL, Castro RD et al. Temporomandibular disorder is more prevalent among patients with primary headaches in a tertiary outpatient clinic. Arq neuropsiquiatr. 2015;73(1 1):913-917. doi: 10.1590/0004-282X20150145

22. Mello VVC, Barbosa ACS, Morais MPLA, Gomes SGF, Vasconcelos MMVB, Caldas Júnior AF. Temporomandibular disorders in a sample population of the Brazilian northeast. Braz Dent J. 2014;25(5):442-446. doi: 10.1590/0103$\underline{6440201302250}$

23. Camacho JGDD, Oltramari-Navarro PVP, Navarro RL, Conti ACCF, Conti MRA, Marchiori LLM et al. Signs and symptoms of Temporomandibular Disorders in the elderly. CoDAS. 2014;26(1):76-80. doi: 10.1590/S231717822014000100011

24. Rani S, Pawah S, Gola S, Bakshi M. Analysis of Helkimo index for temporomandibular disorder diagnosis in the dental students of Faridabad city: A cross-sectional study. J Indian Prosthodont Soc. 2017;17(1):48-52. doi: 10.4103/09724052.194941
25. Chaves TC, Oliveira AS, Grossi DB. Principais instrumentos para avaliação da disfunção temporomandibular, parte I: índices e questionários; uma contribuição para a prática clínica e de pesquisa. Fisioter Pesqui. 2008;15(1):92-100. doi: $10.1590 /$ S1809-29502008000100015 